\title{
ON REAL OR INTEGRAL SKEW LAPLACIAN SPECTRUM OF DIGRAPHS
}

\author{
S. Pirzada, Hilal A. Ganie and Bilal A. Chat
}

Abstract. For a simple connected graph $G$ with $n$ vertices and $m$ edges, let $\vec{G}$ be a digraph obtained by giving an arbitrary direction to the edges of $G$. In this paper, we consider the skew Laplacian matrix of a digraph $\vec{G}$ and we obtain the skew Laplacian spectrum of the orientations of a complete bipartite graph, complete split graph and the join of two graphs. We prove that deleting an edge of a Hamiltonian path in a transitive tournament does not effect the skew Laplacian spectrum. We show the existence of various families of skew Laplacian integral digraphs.

Mathematics subject classification (2010): 05C12, 05C30, 05C50, 15A18.

Keywords and phrases: Digraph, skew Laplacian matrix, skew Laplacian spectrum.

\section{REFERENCES}

[1] C. Adiga, R. Balakrishnan And W. So, The skew energy of a digraph, Linear Algebra Appl. 432 (2010) 1825-1835.

[2] B. A. Chat, H. A. Ganie And S. Pirzada, Bounds for the skew Laplacian spectral radius of oriented graphs, Carpathian J. Math. 35 (1) (2019) 31-40.

[3] D. CvetKovic, M. Doob And H. Sachs, Spectra of Graphs-Theory and Application, Academic Press, New York, 1980.

[4] Q. CAI, X. Li And J. Song, New skew Laplacian energy of simple digraphs, Trans. Combin. 2, 1 (2013) 27-37.

[5] H. A. GANIE, Bounds for the skew Laplacian(skew adjacency) spectral radius of a digraph, Trans. Combin. 8, 2 (2019) 1-12.

[6] Hilal A. GANiE AND S. PirZAda, On the bounds for signless Laplacian energy of a graph, Discrete Appl. Math. 228 (2017) 3-13.

[7] Hilal A. Ganie, A. M. Alghamdi And S. Pirzada, On the sum of the Laplacian eigenvalues of a graph and Brouwer's conjecture, Linear Algebra Appl. 501 (2016) 376-389.

[8] Hilal A. Ganie, Bilal A. Chat And S. Pirzada, Signless Laplacian energy of a graph and energy of a line graph, Linear Algebra Appl. 544 (2018) 306-324.

[9] Hilal A. Ganie, Bilal A. Chat And S. Pirzada, Skew Laplacian spectra and skew Laplacian energy of digraphs, Kragujevac J. Maths. 43 (1) (2019), 87-98.

[10] H. A. Ganie, S. Pirzada, B. A. Chat AND X. Li, On skew Laplacian spectrum and energy of digraphs, Asian-European J. Math. doi:10.1142/S1793557121500510.

[11] Y. P. Hou AND A. X. FANG, Unicyclic graphs with reciprocal skew eigenvalues property, Acta Math. Sinica (Chinese Series) 57, 4 (2014) 657-664.

[12] Xueyi Huang And QiongXiang Huang, On the Laplacian integral tricyclic graphs, Linear Multilinear Algebra 63, 7 (2015) 1356-1371.

[13] G. Indulal, R. BALAKRISHNAN AND A. AnURAdHa, Some new families of integral graphs, Indian J. Pure Applied Mathematics 45, 6 (2014) 805-817.

[14] X. Li AND H. LiAn, A survey on the skew energy of oriented graphs, arXiv:1304.5707v6 [math.CO] 18 May 2015.

[15] S. F. LU AND J. Y. Zou, The new class of Laplacian integral graphs, Advanced Materials Research, 989-994 (2014) 2643-2646.

[16] S. PirZADa AND Hilal A. GANiE, On the Laplacian eigenvalues of a graph and Laplacian energy, Linear Algebra Appl. 486 (2015) 454-468. 
[17] S. PIRZADA, An Introduction to Graph Theory, Universities Press, Orient BlackSwan, India, 2012.

[18] B. Shader AND W. So, Skew spectra of oriented graphs, Electron. J. Combin. 16 (2009) N32.

[19] Y. WANG AND B. ZHOU, A note on skew spectrum of graphs, Ars Combin. 110 (2013) 481-485.

[20] G. XU, Some inequalities on the skew-spectral radii of oriented graphs, J. Inequal. Appl. (2012) 2012:211.

[21] G. Xu AND S. Gong, On oriented graphs whose skew spectral radii do not exceed 2, Linear Algebra Appl. 439 (2013) 2878-2887.

[22] L. You, M. Yan, W. So AND W. XI, On the spectrum of an equitable quotient matrix and its application, Linear Algebra Appl. 577 (2019) 21-40. 